\title{
L'Histoire amoureuse des Gaules au carrefour des genres mondains
}

\author{
Nathalie Freidel \\ Wilfrid Laurier University
}

L'histoire du roman satirique de Bussy-Rabutin est celle d'une récidive : exilé d'abord pour avoir participé aux dissipations d'une jeunesse insolente, le jeune libertin aggrave son cas en composant un tableau de la France galante dont la publication subreptice entraîne son emprisonnement puis sa disgrâce à vie. Celle-ci n’a pourtant pas empêché le grand succès de ce roman inachevé, qui a suscité de nombreuses suites et réécritures ${ }^{1}$. Coralie Robin, qui met en évidence sa structure éclatée et déroutante, divisée en quatre histoires dont les deux dernières s'insèrent dans un récit-

${ }^{1}$ En particulier La France galante [1695], Cologne, P. Marteau. 
cadre, qualifie le roman rabutinien de «monstre formel » (2006, p. 266). Véritable répertoire des genres mondains, l'œuvre de Bussy se présente en effet comme une collection d'entretiens amoureux, de portraits, de lettres, sans oublier les chansons, les bouts-rimés et les sonnets insérés dans la trame du roman, dans lesquels on a pu voir un héritage de la satire ménipéenne (Woodrough, 1988). Quoique Bussy ait revendiqué, dans sa lettre apologétique à Saint-Aignan², l'appartenance de son œuvre au genre romanesque, il est fort probable que les contemporains, dont beaucoup se sont reconnus avec fureur sous les traits des protagonistes de l'histoire ${ }^{3}$, l'aient plutôt perçue comme un recueil juteux de ragots, d'intrigues et d'affaires mettant en cause des personnalités connues de la cour et de la ville (Woodrough, 1991).

Nous sommes donc confrontés à un texte aux contours génériques flous, pur produit d'une culture de la divulgation et de la médisance (voir Darnton) qui s'épanouit dans une littérature marginale - gazettes, journaux, libelles, almanachs, annales - et qui se donne pour tâche la publication incessante de nouvelles de toutes sortes, du bottin mondain à la révélation scandaleuse, en réponse aux attentes d'un public avide de ce genre de révélations. Pour contrer la fadeur ampoulée de l'histoire officielle, on se passionne pour les intrigues et les dessous de cartes, les coulisses du théâtre du monde : " A-t-on publié toutes les galanteries qui se sont faites dans la vieille cour et saura-t-on toutes celles qui se font aujourd'hui ?", s'exclame Segrais dans la préface des Nouvelles françaises (1656, p. 34). Le roman de Bussy dresse le tableau d'une société

\footnotetext{
${ }^{2}$ La lettre figure dans les «Annexes » de l'édition de l'Histoire amoureuse des Gaules que nous prenons pour référence, p. 237.

3 C'est entre autres le cas de la cousine de Bussy, Mme de Sévigné, avec qui la publication de l'Histoire amoureuse entraînera un contentieux durable.
} 
dans laquelle le colportage des nouvelles, la médisance et le goût du scandale sont généralisés.

Dans ce contexte, le genre épistolaire apparaît comme un medium privilégié, ressort essentiel dans la mécanique du jeu amoureux, au point que l'Histoire amoureuse prend par moments des allures de roman épistolaire. De la lettre, fleuron de la culture galante, les protagonistes du roman rabutinien font en effet un usage intensif et subversif, par le brouillage systématique des espaces de la réception. Il semble enfin que, à travers l'écheveau des intrigues et le feuilleton amoureux, ce «roman intrinsèquement polémique» (Robin, 2006, p. 270) s'applique au dynamitage systématique des conventions du loisir mondain, tous genres confondus, en se livrant à une perversion des codes et en remettant en question le système de valeurs et de discours de la société galante.

\section{Une culture de la divulgation}

À bien des égards, l'Histoire amoureuse de Bussy témoigne de la fascination de toute une société pour ce que dissimule le voile des bienséances qu'elle s'est imposé. La publication du privé se décline ainsi en toute une gamme d'attitudes - indiscrétions, confidences, révélations, curiosité, espionnage collectif - et de formes d'écriture - lettres, chansons, sonnets et autres formes poétiques brèves, portraits, etc. La médisance, la raillerie, la calomnie accompagnent souvent ces sortes de lynchages médiatiques. C'est ce qu'on pourrait appeler le côté tabloïd de la littérature galante, qui explique que, parfois, ses cibles s'estiment humiliées et trahies, «livrée[s] entre les mains des 
brigands », selon l'image évocatrice employée par Mme de Sévigné (tome I, p. 101).

Le roman de Bussy met en scène des personnages aisés à reconnaître derrière des pseudonymes transparents, souvent de simples anagrammes, et qui ont fait parler d'eux en leur temps, comme en témoignent Mlle de Montpensier, Tallemant, Saint-Évremont, Mme de Sévigné ou Saint-Simon, dont les anecdotes et les portraits viennent souvent compléter ceux de Bussy. On évolue dans une sphère mi-privée mi-publique, peuplée de gens de diverses conditions, où le jeu des intrigues aplanit les hiérarchies: "Une femme, qui avait aimé des bourgeois et des gentilhommes, pouvait bien aimer un beau prince » (p. 76). Les acteurs de l'histoire, que l'auteur a côtoyés et qui faisaient, pour certains, partie du cercle de ses intimes, constituent une jeunesse dorée mais tumultueuse et emportée, qui compense son peu d'enthousiasme pour la gloire des armes par des conquêtes féminines dans lesquelles les jeunes gens s'investissent comme pour une campagne militaire. Les tractations qui suivent la fin de la Fronde, dans l'« Histoire d'Angélie », signalent à la noblesse qu'elle devra désormais rechercher la gloire sur un autre terrain.

Puisqu'il n'est plus question de publier la gloire des héros, ni de conter leurs périlleuses aventures, le narrateur se propose d'offrir au public, sur un mode héroï-comique, le récit de leurs exploits amoureux. "Les dames donnent de l'estime aussi bien que les armes»(p.49), expliquent au prince de

\footnotetext{
${ }^{4}$ Par exemple, le duc de Candaule, avec qui il a servi dans l'armée et dont il dit dans ses Mémoires : "Il n'y avait guère de secret dont il ne me fit confidence. » (p. 30, note 3) Bussy connaissait aussi très bien le chevalier de Gramont pour avoir servi un temps avec lui sous Turenne et Condé.
} 
Marsillac des amis soucieux de sa réputation. Comme il allait jadis faire ses armes, le jeune premier se lance désormais à l'assaut de la citadelle féminine " pour faire parler de lui ». La trame militaire, qui doublait les intrigues amoureuses dans les romans héroïques, est presque entièrement évacuée, hormis quelques allusions, pour laisser place à une succession d'aventures galantes, centrées sur cinq figures féminines : la comtesse d'Olonne, Mme de Fiesque, Mme de Chatillon, Mme de Sévigné, Mme de Montglas. Le roman de Bussy comporte donc une double visée satirique, prenant à rebours le roman héroïque d'une part et les conventions mondaines d'autre part. C'est en définitive toute l'entreprise d'autopromotion de la société galante (Denis, 2001) qui vole en éclat sous une plume qui annonce les traits mordants de Tallemant, les portraits au vitriol de Saint-Simon, et même les savoureuses caricatures de Proust.

Bussy souligne les paradoxes d'une culture selon laquelle dissimulation rime avec ostentation. À quoi bon le secret, la discrétion et la pudeur requis par la bienséance alors que «de véritables institutions diffusent inlassablement des nouvelles sur tout et sur tous » (Muchembled, 1986, p. 307) ? Comment concilier cette obsession de la divulgation avec la hantise de la diffusion? Trimalet éprouve le besoin de s'excuser auprès de son confident d'avoir tardé à diffuser les détails de ses fiascos répétés auprès d'Ardélise : «Quelque indiscret que l'on soit, il n'y a point d'affaire que l'on ne tienne secrète au commencement quand on a pu se passer de confident pour en venir à bout. » (p. 80)

Toute l'action de l'Histoire amoureuse est exposée, comme sur une scène de théâtre, à l'intention d'un public invisible dont 
on entend la rumeur. C'est le «trop de bruit» que fait la beauté d'une femme qui lui amène des soupirants, puis le "grand bruit » que fait la conduite de ces derniers qui attire des rivaux. « Le bruit public » se charge ensuite d'apprendre aux uns et aux autres leurs trahisons réciproques, entraînant des querelles qui "font grand bruit», parfois suivies de raccommodements «publics». Au passage, le roman contribue à souligner les incohérences d'un système dans lequel la publicité des aventures amoureuses parfait la réputation d'un homme mais ruine celle d'une femme. Et malheur à celles qui se risquent à se comporter en homme sur ce chapitre. L'indiscrétion d'Ardélise, qui donne au public les lettres de Samilcar, "surprit tout le monde » : « On avait vu jusque là des amants indiscrets, et point encore de maîtresse. » (p. 90)

Bussy ne se contente pas de souligner cette omniprésence du public, il met au jour les rouages de la publication: la perspicacité et la clairvoyance de l'amant, qui fait défaut au mari (p. 28), l'obsession de la réputation, les dérèglements de la passion qui s'expose à la vue de tous, le goût de la médisance et de la raillerie, associées au badinage mondain. L'art de médire de son prochain, quoique condamné par les manuels de savoirvivre, fait partie intégrante de la culture mondaine, et ce fiel est à la source de la réputation de nombre de protagonistes de l'Histoire amoureuse. Du comte de Gramont, que Bussy situe au centre d'un indémêlable écheveau d'intrigues, Saint-Simon dira : "C'était un homme de beaucoup d'esprit, mais de ces esprits de plaisanterie, de réparties, de finesse et de justesse à trouver le mauvais, le ridicule, la faille de chacun, de la peindre en deux coups de langue irréparables et ineffaçables [...]. » (tome II, p. 856) 
De manière générale, il semble que, pour les protagonistes du roman de Bussy, le plus grand intérêt d'une intrigue réside dans l'histoire que l'on va pouvoir en tirer afin d'en réjouir le public. L'amant n'a de cesse de prendre son entourage à témoin, de s'épancher auprès de confidents qui semblent choisis exprès pour leur peu de fiabilité, et de faire perversement étalage de circonstances qu'on pourrait juger peu honorables. Ardélise avoue à son mari «non seulement son amour pour Candale, non seulement qu'elle lui avait accordé les dernières faveurs, mais encore les particularités de ses jouissances » (p. 47). Trimalet, qui découvre avec Ardélise que « ce n'est pas [son] fait que les dames », avoue lui-même que le plus grand plaisir d'une aventure réside dans le récit qu'on en fait après coup: "j'aime assez à conter une aventure amoureuse ». Ainsi confie-t-il ses exploits douteux à Vineville «par le détail le plus exact du monde»(p.80). Quant au chevalier de Gramont, qui décide de s'engager auprès d'Ardélise par jeu, peu lui importe l'issue, « pourvu qu'il pût badiner, faire dire au monde qu'il était amoureux » (p. 56). Ce que la fiction de Bussy nous révèle, c'est donc moins une chronique scandaleuse qu'une culture de la divulgation, qui s'épanouit dans quelques formes littéraires privilégiées, au cœur de la sphère mondaine.

\section{Un roman épistolaire?}

Bernard Bray a mis en évidence, dans l'Histoire amoureuse, une typologie du discours amoureux, tantôt oral, sous forme de conversations, tantôt écrit, sous forme de lettres (trente-huit au total) de longueur et de style variés, pastichant toutes les 
situations épistolaires possibles. Or, tous les soupçons pèsent sur la lettre, symbole de l'art d'aimer et de vivre mondain, devenue dans la fiction de Bussy celle par qui le scandale arrive, en grande partie responsable du désordre amoureux et des intrigues enchevêtrées dont il fait la peinture. Constamment interceptée, recopiée, feinte, détournée, déguisée, elle vaut moins par elle-même que par l'usage compromettant qui en est fait dans un jeu complexe de dénonciations et d'accusations indirectes qui annonce Les Liaisons dangereuses.

C'est en fait tout un système de valeurs et de discours, celui de la belle galanterie, qui est remis en cause par l'utilisation que les protagonistes de l'Histoire amoureuse font de ce bastion de la culture mondaine. Dans les Conversations nouvelles, Mlle de Scudéry déclarait: «Ceux qui reçoivent une belle lettre d'amitié se font honneur en la montrant. » (tome II, p. 538-539) Dans le récit de Bussy, ce principe est radicalement inversé : on montre les lettres non pour se faire honneur, mais au contraire pour déshonorer quelqu'un ou ruiner une réputation. De manière générale, les épistoliers y font de la lettre un usage qui dénature et sape dangereusement les fondements d'un code laborieusement mis en place par les conventions épistolaires. Ce qu'on nomme, entre honnêtes gens, commerce épistolaire, devient, entre Ardélise et ses correspondants, commerce au pied de la lettre, ou plutôt marchandage éhonté. Elle y négocie en effet ses charmes avec Crispin, l'intendant des finances Jacques Paget, contre espèces sonnantes et trébuchantes, avant de déclarer ce correspondant bien plus selon son goût que ceux qui remplissent leurs lettres de serments d'adoration et d'offres de services dont on n'a que faire. Dans les intrigues dont Bussy expose les ressorts, la lettre apparaît comme une monnaie d'échange, une pièce que l'on met 
en gage chez le destinataire, quitte à réclamer ultérieurement son dû une fois le contrat rompu. C'est toute une économie parallèle de la lettre qui vient subvertir et parodier un code préétabli. Ainsi, la lettre de Candale reprochant à Ardélise ses infidélités renverrait au modèle épistolaire de la lettre de reproche, ou invective, sans le commentaire ironique qui l'accompagne: «il venait de perdre un combat et cela n'avait pas peu contribué à l'aigreur de sa lettre » (p. 42).

Une galanterie est un secret voué à être découvert; sans cette reconnaissance publique, elle n'est rien et pourrait tout aussi bien n'avoir jamais eu lieu. Tallemant se moque de La Tour-Roquelaure, qui prétendait avoir couché avec Mme de Comminges, Mme de Fosseuse et Mme d'Uxelles : «Et qui vous croirait, lui rétorque la comtesse de Fiesque, vous n'avez pas une lettre » (tome II, p. 384). La lettre n'aide donc pas tant les amants à entretenir leur commerce qu'à obtenir du public une forme de reconnaissance sans laquelle ce commerce n'aurait aucune valeur. C'est pourquoi le premier réflexe des destinataires est de «montrer » la lettre reçue à leur entourage immédiat, ce que fait Ardélise en sacrifiant Aigremont (p. 59). Les confidents semblent ainsi nommés par antiphrase, étant fréquemment des rivaux, donc les moins à même de mériter la confiance dont on les gratifie en leur montrant des lettres. Bien plus, Bussy met en scène de véritables faussaires, tel Aigremont / Gramont, qui se plaît à corrompre les valets, intercepter les courriers et contrefaire les écritures : «Il était libéral jusqu'à la profusion et par là sa maîtresse ni ses rivaux ne pouvaient avoir de valets fidèles. » (p.53) Sa rivalité avec Trimalet / Guiche, autre personnage singulièrement dénué de scrupules, est à l'origine d'un invraisemblable embrouillamini épistolaire: fausses lettres, missives soutirées de force, 
interceptées, dérobées, recopiées, falsifiées, etc. Le cumul des amants ajoute à la confusion: les courriers s'entrecroisent, échouent entre les mains d'un autre destinataire, provoquant d'innombrables quiproquos. Le rituel épistolaire, qui avait pu constituer pour toute une génération une école du monde au même titre que l'art de la conversation, dégénère ici en vils combats et rapports de force, dans lesquels l'intérêt personnel passe au premier plan et où tous les coups sont permis. La rupture entre Ardélise et Samilcar / Marsillac est sur ce point exemplaire. Lorsque Samilcar s'aperçoit qu'Ardélise le trompe avec Trimalet, il donne ses lettres à tout le monde, avec pour consigne de les montrer partout. Mais l'amant dépité ne se contente pas de cette publication ignominieuse, il va jusqu'à la délation en montrant à Léonor / Montpensier une lettre de Trimalet à Ardélise « dans laquelle il parlait fort mal de la Reine et de Lycidas [Monsieur]» (p. 88).

On se heurte ici à l'ambiguïté et au double sens de la notion de public au XVII e siècle, «nébuleuse sémantique dominée par l'ombre du pouvoir d'État» (Jouhaud et Viala, 2002 , p. 12). La seule mention des plus hauts personnages de l'État, au milieu de ce que l'on peut bien nommer un chaos spirituel et moral, explique que l'Histoire amoureuse ait pu passer pour un pamphlet attaquant le roi et la famille royale. Dans l'«Histoire d'Angélie », où l'on entend encore très distinctement les échos de la Fronde, la galanterie est encore plus intimement liée aux affaires. L'abbé Baurin, victime d'une intrigue galante, rédige des lettres dans lesquelles il « déchire » Angélie puis, "pour achever sa vengeance », montre à la Reine les lettres les plus enflammées qu'il a reçues de sa maîtresse (p.118). Le narrateur précise à cette occasion n'en rapporter que «les fragments les plus honnêtes », laissant au lecteur la 
liberté d'imaginer le pire. L'atmosphère est nettement plus tendue que dans l'histoire précédente, parce qu'il ne s'agit plus de publier l'intime pour divertir et satisfaire la curiosité d'un public friand d'indiscrétions de ce genre. Cette fois, l'objectif avoué est d'éveiller les soupçons par des révélations scandaleuses et d'attirer sur la victime les foudres du pouvoir. Dès l'instant où les intrigues amoureuses servent de paravent à des complots politiques, comme celui contre Mazarin dans lequel est impliquée Angélie, et mettent en cause de hauts personnages, comme le roi d'Angleterre, la publication de la vie privée a un tout autre retentissement que lorsqu'on évoluait dans les cercles de la bourgeoisie financière.

La fin de l' «Histoire d'Angélie » marque l'apogée du quiproquo épistolaire. Ardélise convient avec Chamuy que seules ses lettres "souscrites d'un double sceau» seraient dignes de foi. Mais Chamuy «supprime la lettre d'intelligence » et envoie à Condé celle que Foucquet avait écrite (p. 130). Làdessus, Foucquet ayant surpris «une lettre fort tendre» (p. 131) d'Angélie à Condé, celle-ci entreprend d'écrire à chacun de ses amants, mais à la suite d'une méprise, on remet à l'un la lettre destinée à l'autre et vice versa. L'«équivoque » (p. 133) pousse Angélie au désespoir, si bien qu'elle se risque à s'introduire de nuit chez Foucquet pour reprendre et détruire ses lettres. On comprend, en voyant les protagonistes réduits à de telles extrémités, qu'il ne s'agit plus seulement pour eux de défendre leur honneur mais de faire face à une menace de représailles, révélatrice d'une atmosphère de répression latente. Il semblerait que le romancier se livre ici au récit anticipé - et mis en abyme - de la publication de son texte. 


\section{Le crépuscule des genres mondains}

À y regarder de plus près, la fiction de Bussy constitue une étonnante mise en abîme de sa réception, comme si le scandale survenu lors de la diffusion imprimée était déjà inscrit au cœur du roman. L'auteur de l'Histoire amoureuse va en effet être confronté à tous les malentendus, les soupçons infondés et les condamnations auxquels donnent lieu les indiscrétions de ses protagonistes, en particulier à l'égard de la chose écrite. Luimême recopié, peut-être remanié, diffusé puis imprimé clandestinement, livré enfin à l'ire du souverain, le manuscrit de Bussy va subir précisément le sort des missives de son histoire.

Le scandale provoqué par la publication de l'Histoire amoureuse symbolise la fin d'une culture dans laquelle « publier » se limitait à révéler devant un public restreint, dans une conversation ou dans une lettre. En dépit du mépris mondain, la domination de l'imprimé va bientôt s'imposer définitivement dans le domaine des Lettres et toute œuvre devra s'y soumettre pour être reconnue comme telle. Dès lors, la publication signifiera une inévitable «vulgarisation », dont le système d'Ancien Régime a cherché si longtemps à se préserver. Les rois permettaient qu'on eût « de petits mots joyeux avec eux à part [...] mais ne voulaient que le vulgaire en fût abreuvé », constatait Brantôme dans Les Dames galantes (1566, p. 467). Or le roman rabutinien, qui décrit et met en scène - en particulier, on l'a vu, à travers l'usage de la lettre - un nouveau régime de diffusion de l'écrit, remet en cause ce règne de l'aparté. L'absence de distinction entre officieux et officiel, réel et fiction, privé et public autorise des glissements continuels, qui expliquent qu'un manuscrit à usage rigoureusement 
confidentiel soit tombé dans le domaine public. Mêlant galants de ville et galants de cour, sans parler d'un certain nombre de personnages amphibies, l'Histoire amoureuse se situe dans un passé encore d'actualité. Les pseudonymes choisis par Bussy étaient sans nul doute transparents pour les contemporains. Beaucoup sont de simples anagrammes, déchiffrables par le premier venu. Nul besoin de faire partie d'une coterie pour reconnaître la comtesse de Fiesque dans Fésique, Marsillac en Samilcar, Villars en Larisse, Jarzé en Zérige, etc. D’autres noms ne sont pas déguisés, comme Manicamp, ou à peine modifiés, comme Castillante pour Jeanin de Castille ou Foucqueville pour Foucquet. Les rares recours à la tradition romanesque ${ }^{5}$ sont si isolés qu'ils font plutôt figure d'anomalie et produisent un effet de discordance burlesque, soulignant le côté factice de la façade héroïque. Il y a là une rupture décisive avec la tradition du roman héroïque et galant, qui a conduit Jacques Prévot à voir dans l'Histoire amoureuse "un anti-Cyrus ou une anti-Clélie » (1969, p. 10).

Enfin et surtout, Bussy se met lui-même en scène dans la dernière et la plus problématique «partie » de l'Histoire, où le badinage mondain fait place au libertinage. L'apparition du « je » de l'auteur, dans le portrait de Mme de Cheneville, achève de brouiller les frontières entre privé et public, entre fiction et réalité. Le lecteur se trouve dès lors confronté à un objet littéraire non identifié, qui tient de la gazette, des mémoires et de la nouvelle tout en reprenant encore les codes de la littérature mondaine : histoire intercalée, utilisation de la lettre et du portrait, usage des pseudonymes, etc. Mais l'intention

5 Orondate figure dans Cassandre de La Calprenède (1642-1645) ainsi que dans Oroondate ou Les amans discrets de Guyon Guérin de Bouscal (1647). 
ouvertement parodique vient miner ce code de l'intérieur. De la même manière que les quatre compagnons de Roissy se livrent à une parodie de la dévotion, célébrant des Pâques à rebours et faisant des contresens du discours chrétien de la retraite, Bussy fait un usage paradoxal de la rhétorique mondaine.

C'est en fin de compte toute la «poétique du loisir mondain » (Génétiot, 1997) qui est foulée aux pieds par le cercle impénitent des quatre amis, qui n'est pas sans évoquer les compagnies choisies de La Fontaine. Le décor champêtre, la partie de chasse et la promenade servent de cadre à un échange qui parodie insolemment tous les principes de la conversation mondaine. Le narrateur prend ici à contre-pied les règles établies dans les manuels de civilité ou dans le chapitre du Grand Cyrus consacré à la raillerie. À l'élégance, la grâce, la mesure et l'à-propos prônés par les théoriciens de l'honnêteté, Bussy substitue le sarcasme, le persiflage, l'ironie mordante et la médisance. Il s'agit, selon les propres termes des protagonistes, de "médire de tout le genre humain » après en avoir préalablement excepté quelques rares «bons amis». Le comble de l'hétérodoxie est atteint lorsque Bussy découvre au matin Manicamp dans le lit de Trimalet. L'homosexualité, latente dans l'univers des bergeries, se trouve ici justifiée dans un contexte de relativisme moral qui ne manque pas de piquant: «Pour moi, je ne condamne point vos manières; chacun se sauve à sa guise.»(p.151) Suivent les fameux "Alléluias », d'attribution incertaine il est vrai, dont la virulence blasphématoire n'épargne pas la famille royale.

Derrière les attaques ad hominem, c'est en fin de compte la culture galante dans son ensemble qui est visée, à travers les genres littéraires qu'elle a mis à la mode. Si l'on y regarde de 
près, le portrait de Mme de Cheneville (p. 152-164), que Mme de Sévigné a jugé si offensant, vise moins la personne que la gaieté factice, le goût de la flatterie, l'obsession galante, l'hypocrisie morale, la servilité politique qui caractérisent la sociabilité mondaine. L'hypocrisie et la fausseté des prétendues «bienséances » éclatent dans l'usage systématique du chiasme — « un sot éveillé l'emportera toujours auprès d'elle sur un honnête homme sérieux » - , de l'antithèse - «froideur du tempérament / chaleur de l'esprit », accorder « le plaisir avec le monde, le plaisir avec la vertu », " paraître tout ce qu'on n'est pas» - et du paradoxe - «je la trouvai la plus jolie fille du monde pour être femme d'un autre », «la plus forte raison qui m'obligea d'en faire ma maîtresse fut celle qui m'avait empêché d'être son mari », "si elle n'était votre femme, elle serait votre maîtresse ». Que l'on juge tout ce qui sépare cette rhétorique instable de l'art du portrait tel que Segrais, par exemple, le définit dans son Recueil de portraits et éloges : "Ce sont de grands modèles de beauté, de douceur, de piété, de prudence, d'amour, de générosité et de modestie, que l'on donne au public. » (1656, vol. 1, p. VI) Avec le portrait, c'est un pilier de l'esthétique galante qui s'effondré ${ }^{6}$.

L'art du récit selon Bussy est une remise en cause brutale de l'ancien régime de la publication, dans lequel il s'agissait en réalité de faire illusion, de présenter une façade acceptable à un public complice, d'atteindre un consensus. Au contraire, le texte de Bussy est polémique par essence et dénonce la conspiration galante, s'adressant désormais à un public anonyme qui ne

\footnotetext{
${ }^{6}$ Boileau, dans son Dialogue des Héros de Romans [1713], œuvre rédigée dans les mêmes années que l'Histoire amoureuse et tout aussi virulente à l'encontre de la culture galante, vise le même résultat au moyen d'un portrait de Tisiphone, qui dénonce les ficelles et les travers du genre (p. 473).
} 
partage plus les valeurs du cercle restreint des initiés. La vérité est le dernier des soucis du public mondain : "on n'a jamais cessé de dire qu'elle l'avait aimé. Et bien que cela ne fut pas vrai, c'était toujours le plus vraisemblable à dire » (p. 163). Bussy, pour sa part, laisse souvent planer le doute, décrit une réalité trouble et laisse au lecteur le soin d'exercer son jugement et de choisir entre différentes versions possibles. L'usage généralisé de la conjonction «soit que, soit que », la juxtaposition du pour et du contre, du positif et du négatif, de l'éloge et de la calomnie, les mouvements binaires supposent un lecteur actif plutôt que complice. La logique du secret partagé et de l'exclusivité, la communauté des valeurs sont remises en cause par la distance que Bussy affiche vis-à-vis de ses personnages. On comprend mieux dès lors l'ampleur du scandale déclenché par la publication de ce roman et le ressentiment durable qu'il a entraîné. Par-delà la médisance et la calomnie à l'égard des particuliers, c'est toute la collectivité mondaine qui s'est sentie visée par une œuvre qui faussait et dénaturait le système de représentations qui avait assuré le triomphe du Parnasse galant.

Le «désordre» qui, selon Coralie Robin, caractérise l'œuvre de Bussy est donc avant tout générique. Il s'agit, en brouillant les frontières entre gazettes et romans, nouvelles et genre épistolaire, fiction et vérité, de désorienter le lecteur attaché à un idéal d'harmonie galante. La plasticité du texte rabutinien, qui accueille la pléiade des petits genres mondains, fait de l'Histoire amoureuse un répertoire poétique autant qu'un recueil d'anecdotes. Désordre galant et amoureux et multiplicité générique finissent par se confondre pour produire une œuvre décalée dont on peut comprendre qu'elle ait été perçue comme une menace par le pouvoir. Toutefois, Bussy ne 
conçoit pas seulement une machine de guerre dirigée contre les genres mondains, il crée également une œuvre expérimentale qui contribue à la rénovation du genre romanesque à un moment charnière, particulièrement difficile à cerner du fait de la confusion des intérêts et de la persistance d'espaces mixtes. Les imbroglios créés par la circulation incontrôlable des lettres, le topos du discours surpris traduisent la conscience des aléas du sens liés à la diffusion imprimée qui conduit le roman vers des lecteurs nombreux, aux aspirations inconnues et peut-être contradictoires, paradoxe constitutif de la destination romanesque, que Jacques Rancière a ainsi formulé : «le romancier écrit pour ceux qui ne devraient pas le lire » (1988, p. 97).

\section{Bibliographie}

Bolleau, Nicolas. (1966), CEuvres complètes, Paris, Gallimard, coll. «Bibliothèque de La Pléiade ».

BrantôME, Pierre de Bourdeille, dit. (1981 [1566]), Les Dames galantes, Paris, Gallimard, coll. « Folio classique ».

BRAY, Bernard. (1995), «L'Écrire ou le dire : l'expression de l'amour dans l'Histoire amoureuse des Gaules de BussyRabutin ", dans Bernard Bray et Christophe Strosetski (dir.), Art de la lettre, art de la conversation à l'époque classique en France, Paris, Klincksieck, p. 245-255.

BuSSY-RAButin, Roger, comte de. (1993 [1665]), Histoire amoureuse des Gaules, éd. Jacqueline et Roger Duchêne, Paris, Gallimard, coll. « Folio classique ». 
DARNTON, Robert. (2010), Le Diable dans un bénitier. L'art de la calomnie en France, 1650-1800, Paris, Gallimard.

DenIS, Delphine. (2001), Le Parnasse galant. Institution d'une catégorie littéraire au XVII e siècle, Paris, Champion, coll. «Lumières classiques ».

GÉNÉTIOT, Alain. (1997), Poétique du loisir mondain de Voiture à La Fontaine, Paris, Champion, coll. « Lumières classiques ».

Jouhaud, Christian et Alain Viala (dir.). (2002), La Publication entre Renaissance et Lumières, Paris, Fayard.

MuCHEMBlED, Robert. (1988), L'Invention de l'homme moderne. Culture et sensibilités en France du XVe au XVIIIe siècle, Paris, Fayard.

PrÉvot, Jacques. (1969), «L'art du portrait chez BussyRabutin », Revue d'Histoire Littéraire de la France, ${ }^{\circ}{ }^{\circ}$, janv.fév., p. 1-12.

RANCIÈRE, Jacques. (1988), La Parole muette. Essai sur les contradictions de la littérature, Paris, Hachette.

RoBIN, Coralie. (2006), "L'Histoire amoureuse des Gaules de Bussy-Rabutin: cantique de la médisance ou machine de guerre ? », Littératures Classiques, n 59, p. 265-282.

SAINT-Simon, Louis de Rouvroy, duc de. (1983 [1856]), Mémoires, éd. Y.Coirault, Paris, Gallimard, coll. « Bibliothèque de La Pléiade ».

SCUDÉRY, Madeleine de. (1684), Conversations nouvelles sur divers sujets, dédiées au Roy, Paris, Barbin. 
SEGRAIS, Jean Regnault de. (1656-1657), Les Nouvelles françaises ou les divertissements de la Princesse Aurélie, Paris, A. de Sommaville.

-. (1659), Recueil de portraits et éloges, Paris, Sercy-Barbin.

SÉvigné, Marie de Rabutin-Chantal, marquise de. (1972-1978 [1734]), Correspondance, Paris, Gallimard, coll. « Bibliothèque de la Pléiade ».

Tallemant des Réaux, Gédéon. (1960 [1834]), Historiettes, Paris, Gallimard, coll. « Bibliothèque de La Pléiade».

Woodrough, Elisabeth. (1988), «Bussy et la satire ménipéenne », Rabutinages, $\mathrm{n}^{\circ} 3$.

—. (1991), "L'Histoire amoureuse des Gaules, satire de cour », Rabutinages, $\mathrm{n}^{\circ} 6$.

Zoberman, Pierre. (1992), "Entendre raillerie", Thèmes et genres littéraires au XVII et XVIII e siècles, Mélanges offerts à Jacques Truchet, Paris, Presses universitaires de France.

\section{Résumé}

En brouillant les frontières entre gazettes et roman, nouvelles et genre épistolaire, fiction et Mémoires, l'Histoire amoureuse des gaules de Bussy-Rabutin apparaît comme un "monstre formel », dont la publication scandaleuse rompt l'harmonie du Parnasse galant. Prenant à rebours les conventions du roman héroïque aussi bien que de la politesse mondaine, Bussy semble anticiper sur le toast porté par Swann «À la malveillance ! son roman satirique met en scène une culture de la divulgation qui 
se raffine en art de la médisance par le détournement et la perversion des usages. Ainsi la lettre, fleuron de l'idéal des civilités, devient-elle, entre les mains de protagonistes sans scrupules, l'instrument même du renversement des valeurs. Devant une telle entreprise de perversion systématique des règles de la morale aussi bien que de la poétique mondaine, on peut à bon droit se demander si Bussy ne sonne pas le glas d'une culture galante enracinée dans les terres utopiques de Confidences et Discrétion.

\begin{abstract}
If Bussy-Rabutin's novel, the Histoire amoureuse des Gaules, caused such a scandal upon its clandestine publication, one should not be surprised, considering this very atypical text ignores all boundaries and rules, not only in terms of mundane but also literary canons. Its mixing of styles, genres and structures, between "gazettes", novel, epistolary writing and "mémoires" makes it appear as a formal monster and unwanted intruder in the sphere of triumphant gallantry. Subverting conventions of the heroic and the gallant, Bussy appears as a precursor of Swann and his homage, in Proust's Recherche, "to malevolence". His satirical novel reveals a culture of divulgation, gossiping and slandering, excelling in perverting its own conventions and the ideal of civilities. The art of letter writing, considered as the ultimate sociability school, turns in Bussy's outrageous stories into the instrument of a massive subversive plot. Such systematic ways of perverting poetical as well as social values make the Histoire amoureuse a decisive weapon against the all-powerful gallantry, deeply rooted in the XVIIth century system of representations.
\end{abstract}

